152

\title{
AUTOANTICORPI E NEUROPATIE
}

'Ciarrocchi G.; 'Neri M.A.; 'Rondello G.; 'Tocchini M.; ${ }^{2}$ Logullo F.; ${ }^{2}$ Manicone M.G.; ${ }^{2}$ Provinciali L.; ${ }^{3}$ Derioni C.

U.O. Laboratorio analisi, Ospedali Riuniti - Ancona; ${ }^{2}$ U.O. Clinica di Neurologia, Università degli studi Ancona; ${ }^{3}$ Research Scientific Service, Alifax Diagnostics Padova.

Introduzione. Anticorpi rivolti verso componenti glicoconiugati dei nervi periferici sono stati associati ad una varietà di neuropatie sensitive e/o motorie.

In particolare, anticorpi anti-gangliosidi (GM1, GQ1b, GT1, ecc.) ed anti-glicoproteina associata alla mielina (MAG) sono stati individuati nel siero di soggetti con diversi tipi di neuropatia a sospetta eziologia autoimmune.

L'evidenza di una correlazione tra produzione di autoanticorpi e danno selettivo dei componenti neuronali è stata in molti casi ben dimostrata, mentre è risultata carente in altri. Scopo. Verificare l'associazione tra presenza di anticorpi verso differenti antigeni mielinici e il quadro clinico ed elettromiografico-elettroneurografico.

Materiali e Metodi. Nel periodo febbraio 2004-aprile 2005, sono stati eseguiti 400 test complessivi su campioni di siero per la ricerca di anticorpi anti-MAG $(\mathrm{n}=71)$, anti-Gangliosidi $(\mathrm{n}=89)$, anti-Neuronali HU $(\mathrm{n}=80)$, YO $(\mathrm{n}=80), \mathrm{Ri}(\mathrm{n}=80)$. A tale scopo sono stati impiegati test-kit basati sui metodi immunofluorescenza (IFI) per anti-MAG, IMMCO Diagnostic; Western-Blot (WB) per anti-MAG e anti-Neuronali, ZEN TECH Diagnostic; Dot-Blot (DB) per anti-Gangliosidi, Alphadia Diagnostic (Belgio); tutti i kit sono stati forniti da ALIFAX Italia.

Risultati. La ricerca di anticorpi anti-MAG è risultata positiva al test IFI in 16 campioni, con evidenza clinica di polineuropatia demielinizzante cronica in 14/16; 1 caso non presentava allo stato segni clinici risolutivi, pur in presenza di IgMk MGUS; 1 caso era associato a gammopatia biclonale IgG e IgMk MGUS. Il test WB confermò 15/16 casi positivi, con riscontro di banda antigenica p100 di MAG. Positività per anticorpi anti-Gangliosidi sono stati riscontrati in 8 sieri. La differente tipologia anticorpale riscontrata era rivolta verso antigeni gangliosidici GM1, GT1a, GT1b, GD1b, GQ1b, GD3, Sulfatidi, variamente associati a neuropatia motoria multifocale, multineuropatia sensitivo-motoria di tipo demielinizzante in M.di Waldestrom, sindrome di Guillain-Barré.

Conclusione. I dati preliminari dello studio sembrano confermare la correlazione dei dati sierologici con quadri clinici ed elettrofisiologici rilevanti dal punto di vista eziologico con importanti ricadute terapeutiche.

Gli anticorpi anti-MAG ed alcuni tra gli anti-Gangliosidi appaiono un utile presidio nel comporre il complesso puzzle diagnostico.

La prosecuzione dello studio metterà in risalto aspetti della qualità analitica dei test (sensibilità, specificità) ed altri relativi alla strategia terapeutica ed al follow up. 